\title{
Inmunonutrición perioperatoria. Consenso de la Asociación Colombiana de Nutrición Clínica
}

\author{
Perioperative Immunonutrition. \\ The Consensus of the Colombian Clinical Nutrition Association
}

\author{
Charles Bermúdez ${ }^{1 *}$, Álvaro Sanabria ${ }^{2}$, Juan Ayala ${ }^{3}$, Andrés Becerra ${ }^{4}$, Mauricio Chona ${ }^{5}$, Liceloth Colpas ${ }^{6}$, \\ Ana Henao ${ }^{7}$, Iván Katime ${ }^{8}$, Lina López ${ }^{9}$, Luis Marroquín ${ }^{10}$, Ricardo Merchán ${ }^{11}$, Martha Muñoz ${ }^{12}$, \\ Ángela Navas ${ }^{13}$, Justo Olaya ${ }^{14}$, Angélica Pérez ${ }^{15}$, Fernando Pereira ${ }^{16}$, Milena Puentes ${ }^{17}$, \\ Mario Rodríguez ${ }^{18}$, Luisa Torres ${ }^{19}$, Arturo Vergara ${ }^{20}$.
}

Recibido: 1 de febrero de 2019. Aceptado para publicación: 12 de marzo de 2019

Publicado en línea, marzo 23 de 2019

https://doi.org/10.35454/rncm.v2n1.055

\section{Resumen}

Introducción: la inmunonutrición es una alternativa en pacientes quirúrgicos por sus ventajas clínicas. Se pretende unificar conceptos y desarrollar un documento de acceso y consulta fácil en relación con el uso de inmunonutrición en pacientes quirúrgicos en Colombia.

Metodología: se seleccionó un grupo interdisciplinario conformado por 21 expertos en nutrición clínica quienes, previa revisión de la literatura, participaron en la Reunión de Consenso. El análisis y la discusión se realizó en torno a 15 preguntas clasificadas en tres tipos: A. Fundamentos de la utilización de la inmunonutrición, B. Indicaciones, seguridad y beneficios de la inmunonutrición, C. Administración de la inmunonutrición.

Resultados: entre los resultados que obtuvieron consenso se encuentran: el uso de inmunonutrientes como arginina, nucleótidos, glutamina, ácidos grasos omega-3, antioxidantes y simbióticos. La inmunonutrición tiene mayor beneficio en los pacientes quirúrgicos, los pacientes malnutridos que van a ser llevados a cirugía y que sufren trauma mayor y quemaduras graves. No se encontró beneficio de la inmunonutrición en pacientes bien nutridos que van a ser llevados a cirugía electiva, cardiaca, o en pacientes con sepsis severa. Se debe usar 5 - 7 días antes de la cirugía y hasta 10 días después, de preferencia por vía oral/enteral.

Conclusión: existen recomendaciones para el uso de inmunonutrición que son ampliamente aceptadas, las cuales deben orientar la toma de decisiones y el uso de recursos en el sistema de salud colombiano.

Palabras clave: cirugía, inmunonutrición, nutrición artificial, cuidado nutricional.

1. Grupo de Soporte Nutricional, Clínica la Colina y Clínica del Country, Bogotá, D.C., Colombia.

2. Departamento de Cirugía. Universidad de Antioquia. Fundación Colombiana de Cancerología - Clínica Vida, Medellín, Colombia.

3. Grupo de Soporte Nutricional Clínica Los Nogales, Bogotá, D.C., Colombia.

\section{Summary}

Introduction: Immunonutrition is an alternative for surgical patients due to its clinical advantages. The aim is to unify the concepts and develop a document of easy access and quick consultation about immunonutrition use in surgical patients in Colombia.

Materials and methods: An interdisciplinary group of 21 clinical nutrition experts was selected who after reviewing the literature, participated in the consensus meeting. Discussion and analysis were around 15 questions, which they classified into three types: A. Fundamentals of the use of immunonutrition, B. Indications, safety and benefits of immunonutrition, C. Administration of immunonutrition.

Outcomes: Among consensus outcomes, they proposed: the use of immunonutrients such as arginine, nucleotides, glutamine, omega-3 fatty acids, antioxidants and synbiotics. Immunonutrition greatest benefit was for surgical patients, malnourished patients undergoing to trauma surgery and severe burn patients. There was no benefit of immunonutrition for wellnourished patients undergoing elective surgery, severe sepsis or heart surgery. Immunonutrition must be used 5-7 days before surgery and up to 10 days later, preferably orally/enterally.

Conclusion: There are widely accepted recommendations for immunonutrition use. These recommendations should be useful for decision-making and for rational use of resources in healthcare system.

Keywords: Surgery; Immunonutrition; Artificial nutrition; Nutritional care.

\footnotetext{
4. Grupo de Soporte Nutricional Hospital San Vicente Fundación, Medellín, Colombia.

5. Grupo de Soporte Nutricional Clínica Universitaria Colombia. Bogotá, D.C., Colombia.

6. Grupo de Soporte Metabólico en Clínica Nuestra y UCI del Caribe, Servicio
} de Cirugía Clínica Estrios y Somedyt, Cartagena, Colombia. 
7. Grupo de Soporte Nutricional Hospital Militar Central. Bogotá, D.C., Colombia.

8. Grupo de Soporte Nutricional Hospital Militar Central. Bogotá, D.C., Colombia.

9. Grupo de Soporte Nutricional Clínica Universitaria Colombia. Bogotá, D.C., Colombia.

10.Grupo de Soporte Nutricional, Clínica Cardiovascular, Soacha, Colombia.

11.Grupo de Soporte Nutricional, Clínica Colombia, Bogotá, D.C., Colombia.

12.Grupo de Soporte Nutricional Hospital General de Medellín, Colombia.

13.Grupo de Soporte Nutricional, Clínica Reina Sofía, Bogotá, D.C., Colombia.

14.Grupo de Soporte Nutricional, Hospital Hernando Moncaleano, Neiva, Colombia.

*Correspondencia: Charles Bermúdez

chebermud@yahoo.com

\section{INTRODUCCIÓN}

La cirugía puede inducir alteraciones graves del sistema inmunitario que provocan una mayor tasa de complicaciones inflamatorias y sépticas postoperatorias ${ }^{(1)}$. La secuencia de cascadas proinflamatorias que conducen a alteraciones en el sistema inmune en los primeros días postoperatorios comienza en el momento de la lesión quirúrgica. Desde la década de los noventa, diversos estudios en nutrición se han centrado en la capacidad de modular la respuesta inmune a la lesión a través de dietas enterales especialmente formuladas ${ }^{(2)}$. Estas fórmulas llamadas inmunonutrición aportan nutrientes como arginina, glutamina y ácidos grasos $\omega 3$ (aceite de pescado) y se han utilizado para alterar la síntesis de eicosanoides, la producción de citocinas y la función inmune con el objetivo de limitar las reacciones inmunes no deseadas después de la lesión quirúrgica ${ }^{(3,4)}$.

Existe gran cantidad de literatura disponible que sugiere las ventajas del uso de la inmunonutrición durante el periodo perioperatorio, de manera específica, mejorando los desenlaces y en consecuencia los costos de la atención ${ }^{(3-6)}$. La utilización de la inmunonutrición en postoperatorio ha mostrado que solo puede mejorar, pero no revertir, la respuesta catabólica e inmunológica posquirúrgica. Por lo tanto, es probable que comenzar la inmunonutrición en el período preoperatorio sea más efectivo porque se mejora el estado nutricional y se reducen las complicaciones postoperatorias ${ }^{(5)}$.

Aunque se ha demostrado que la inmunonutrición regula positivamente la respuesta inmune del huésped, modula la respuesta inflamatoria y mejora la síntesis de proteínas después de la cirugía, su utilización en Colombia aún es controversial. El objetivo de la

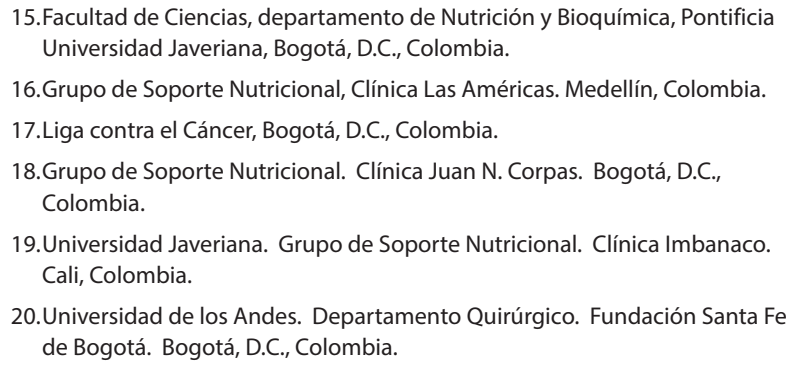

Reunión de Consenso fue proporcionar un análisis de la evidencia científica actualizada, incluidos los antecedentes, las indicaciones, las controversias y sugerencias para futuras investigaciones. Este documento presenta una síntesis del trabajo realizado por el grupo interdisciplinario de expertos y las recomendaciones para el uso adecuado de la inmunonutrición en el sistema de salud colombiano.

\section{METODOLOGÍA}

La Asociación Colombiana de Nutrición Clínica (ACNC) reunió un grupo interdisciplinario de 21 expertos (médicos, nutricionistas, enfermeros y químicos farmacéuticos), para su selección tuvo en cuenta su participación en grupos de soporte nutricional, la profesión y ubicación (ciudad) del sitio de trabajo. Los expertos fueron signados en 5 grupos para responder 14 preguntas correspondientes a 3 temas: A. Fundamentos de la utilización de la inmunonutrición, B. Indicaciones, seguridad y beneficios de la inmunonutrición y C. Administración de la inmunonutrición (Tabla 1). El cuestionario fue realizado por el coordinador del consenso con el apoyo de la Junta Directiva de la ACNC. Se pretendió abordar todos los aspectos relevantes en el uso de Inmunonutrición en el perioperatorio. El cuestionario con las preguntas asignadas fue enviado a cada experto vía correo electrónico.

Durante un periodo de tres meses los expertos realizaron una revisión de la literatura científica y contestaron las preguntas asignadas. Durante la Reunión de Consenso realizada en diciembre 2017 se analizaron y discutieron las 14 preguntas del cuestionario. El presente artículo resume las conclusiones del Consenso. 
Tabla 1. Cuestionario

\begin{tabular}{|c|}
\hline A. Fundamentos de la utilización de la inmunonutrición \\
\hline 1. ¿Cuál es fundamento fisiopatológico que justifica el uso de la inmunonutrición? \\
\hline 2. ¿Cómo/ Cuál es la respuesta inmune a la cirugía? \\
\hline 3. ¿Por qué la inmunonutrición atenúa la respuesta inflamatoria? \\
\hline 4. ¿Qué inmunonutrientes han demostrado beneficio en la administración perioperatoria? \\
\hline B. Indicaciones, seguridad y beneficios de la inmunonutrición \\
\hline 5. ¿Los fórmulas inmunoenriquecidas son seguras en cirugía? \\
\hline 6. ¿En qué pacientes quirúrgicos considera que existe beneficio en el uso de inmunonutrición perioperatoria? \\
\hline 7. ¿En qué pacientes quirúrgicos está contraindicada la inmunonutrición? \\
\hline C. Administración de la inmunonutrición \\
\hline 8. ¿Cuánto tiempo previo a la cirugía se debe iniciar la terapia de inmunonutrición? \\
\hline 9. ¿Cuál debe ser la dosis necesaria para el beneficio planteado en la inmunonutrición preoperatoria? \\
\hline 10. ¿Cuál es la ruta de administración recomendada? \\
\hline $\begin{array}{l}\text { 11. ¿Qué otras medidas no farmacológicas deberían ser usadas para mejorar los resultados cuando se emplea inmunonutrición } \\
\text { preoperatoria? }\end{array}$ \\
\hline 12. ¿Qué factores hacen costo-efectivas las dietas inmunomuduladoras? \\
\hline 13. ¿Qué dificultades existen en Colombia para la implementación de una terapia nutricional como esta? \\
\hline 14. ¿Qué intervenciones reemplazaría el uso de la inmunonutrición en los pacientes en que se demuestre beneficio? \\
\hline
\end{tabular}

\section{1. ¿Cuál es el fundamento fisiopatológico que justifica el uso de la inmunonutrición?}

La cirugía es una modalidad terapéutica en un importante porcentaje de enfermedades. El acto quirúrgico involucra tres actores: el cirujano, el procedimiento quirúrgico y el paciente, los cuales persiguen desenlaces adecuados en términos de baja mortalidad y morbilidad con un impacto positivo en la calidad de vida de los pacientes.

En este sentido el paciente con patología quirúrgica se interpreta como un conjunto de factores modificables y no modificables, que son los que permiten establecer el pronóstico de la cirugía. Los factores no modificables son la edad y las enfermedades de base y los modificables son el riesgo de tromboembolismo, la anemia y el estado nutricional, siendo este último uno de los más importantes ${ }^{(7-9)}$. Sin embargo, la cirugía impone un estrés inflamatorio claramente caracterizado responsable de erradicar microorganismos, promover la adecuada cicatrización y restaurar la hemostasis, que afecta en forma directa el estado nutricional del paciente ${ }^{(10-13)}$.
La relación entre nutrición e inmunidad se reconoce hace más de 30 años y la interacción entre la nutrición y la inmunidad se establece bajo las 3 "I" en nutrición: Infección-Sistema Inmune y Respuesta Inmunológica ${ }^{(13,14)}$. Proporcional a la magnitud del estrés quirúrgico, los requerimientos calóricos, proteicos y de micronutrientes del paciente aumentan, y la malnutrición impacta negativamente la función inmune, en particular la función de los linfocitos $\mathrm{T}$, la actividad del sistema de complemento, la fagocitosis y la quimiotaxis. Esto explica la razón por la cual las intervenciones quirúrgicas en pacientes malnutridos tienen desenlaces inadecuados.

Específicamente, la respuesta inflamatoria al trauma quirúrgico involucra una activación de células del sistema inmune que dependen de sustratos metabólicos tales como arginina, glutamina, ácidos grasos y nucleótidos para su adecuado funcionamiento. Esto implica un gasto acelerado de las reservas y si no se dispone de una fuente permanente, puede afectar la actividad de las células del sistema inmune. Estos sustratos, cuya fuente son los nutrientes, median la activación de las células 
tanto de la respuesta inmune innata, macrófagos, células dendríticas y monocitos, así como las células de la respuesta adaptativa, específicamente linfocitos ${ }^{(6,15)}$.

El reconocimiento de nutrientes claves en la respuesta inflamatoria y su administración en dosis adecuadas permitieron el desarrollo del concepto de farmaconutrición de la mano con la inmunonutrición. Estos términos involucran la administración de estos inmunonutrientes específicos en el periodo perioperatorio.

Por lo tanto, el entendimiento de estos procesos metabólicos permitió que la terapia nutricional migrara de un simple aporte de calorías y proteínas a una intervención terapéutica nutricional que persigue modular la respuesta inmune celular (ver definiciones en Tabla 2) ${ }^{(3,4)}$.

\section{2. ¿Cómo es la respuesta inmune a la cirugía?}

La respuesta al estrés quirúrgico inicia en la liberación de patrones moleculares asociados a daño (DAMPS) que activan la respuesta trimodal de los sistemas: nervioso autónomo, endocrino e inmune (Figura 1). Cuando se da la respuesta innata del sistema inmune por los factores nucleares de transcripción inflamatorios, se producen interleuquinas inflamatorias que estimulan la respuesta adaptativa del sistema inmunológico con predominancia de la respuesta Th2 (antiinflamatoria). En síntesis, la respuesta inflamatoria del estrés quirúrgico iniciada por la liberación de DAMPS, a través de los receptores Toll like activa a este grupo celular anteriormente descrito, que induce a los factores de transcripción nuclear (NF-Kb), ensamblando la respuesta inmune, que busca favorecer un adecuado reparo del daño celular mediante una apropiada cicatrización.

Esta activación y diferenciación celular depende de nutrientes específicos ya mencionados ${ }^{(1,6,8,13)}$.

\section{3. ¿Por qué la inmunonutrición, atenúa la respuesta inflamatoria?}

La inmunonutrición atenúa la respuesta inflamatoria mediante:

\section{a. Control de la activación de factores de transcripción nuclear}

Las respuestas funcionales de todas las células están reguladas por un equilibrio entre las señales estimuladoras y las inhibidoras. Las señales inhibidoras de los linfocitos están mediadas por receptores inhibidores y también por enzimas conocidas como E3 ubicuitina ligasa que marcan ciertas moléculas transmisoras de señales para su degradación, los receptores inhibidores suelen reclutar y activar fosfatasas que contrarrestan las señales inducidas por receptores para el antígeno. Estos receptores contienen estructuras ITIM (immunoreceptor tyrosine-based inactivation motif) en sus colas citoplasmáticas que pueden reclutar fosfatasas con un dominio $\mathrm{SH} 2$ y así atenuar las señales activadoras. En los linfocitos Nk, los receptores inhibidores llamados KIR contienen dominios extracelulares de Ig y estructuras ITIM citosólicas, las tirosinas ITIM pueden ser fosforiladas por cinasas de la familia Src ligadas a la activación del linfocito, además reclutan tirosinas fosfatasas que contienen dominios SH2 llamadas SHIP. La SHP-1 y 2 atenúan las señales iniciadas por la tirosina cinasa de los receptores activadores de los linfocitos NK, así como de los BCR y TCR en los linfocitos B y $\mathrm{T}$ respectivamente. La SHIP elimina fosfatos del PIP3 y de esa manera inhibe la actividad PI3-cinasa en los linfocitos NK y células inmunitarias innatas.

El receptor inhibidor de la familia CD28, CTLA-4 (también llamado CD152) tiene la capacidad de inhi-

Tabla 2. Glosario ${ }^{(10,11,16,36)}$

\begin{tabular}{|l|l|}
\hline Farmaconutriente & Son nutrientes que, a dosis mayores de las fisiológicas, producen un efecto terapéutico. \\
\hline Inmunonutriente & $\begin{array}{l}\text { Es un tipo de farmaconutriente que se espera que, aparte de su efecto nutricional, tenga un efecto } \\
\text { terapéutico sobre el estado inmunológico del paciente (arginina, glutamina, omega-3, nucleótidos). }\end{array}$ \\
\hline $\begin{array}{l}\text { Fórmula enriquecida } \\
\text { con inmunonutrientes }\end{array}$ & Es un producto nutricional en la cual hay una adición de inmunonutrientes. \\
\hline $\begin{array}{l}\text { Producto } \\
\text { fitoterapéutico }\end{array}$ & $\begin{array}{l}\text { Producto medicinal cuyas sustancias activas provienen del material de una planta medicinal o sus } \\
\text { asociaciones, no contiene principios activos aislados y químicamente definidos. }\end{array}$ \\
\hline $\begin{array}{l}\text { Malnutrición } \\
\text { (desnutrición) }\end{array}$ & $\begin{array}{l}\text { Condición que resulta de una combinación de diversos grados de nutrición insuficiente o excesiva y de la } \\
\text { actividad inflamatoria, que conduce a una alteración en la composición corporal y a una función disminuida. }\end{array}$ \\
\hline
\end{tabular}




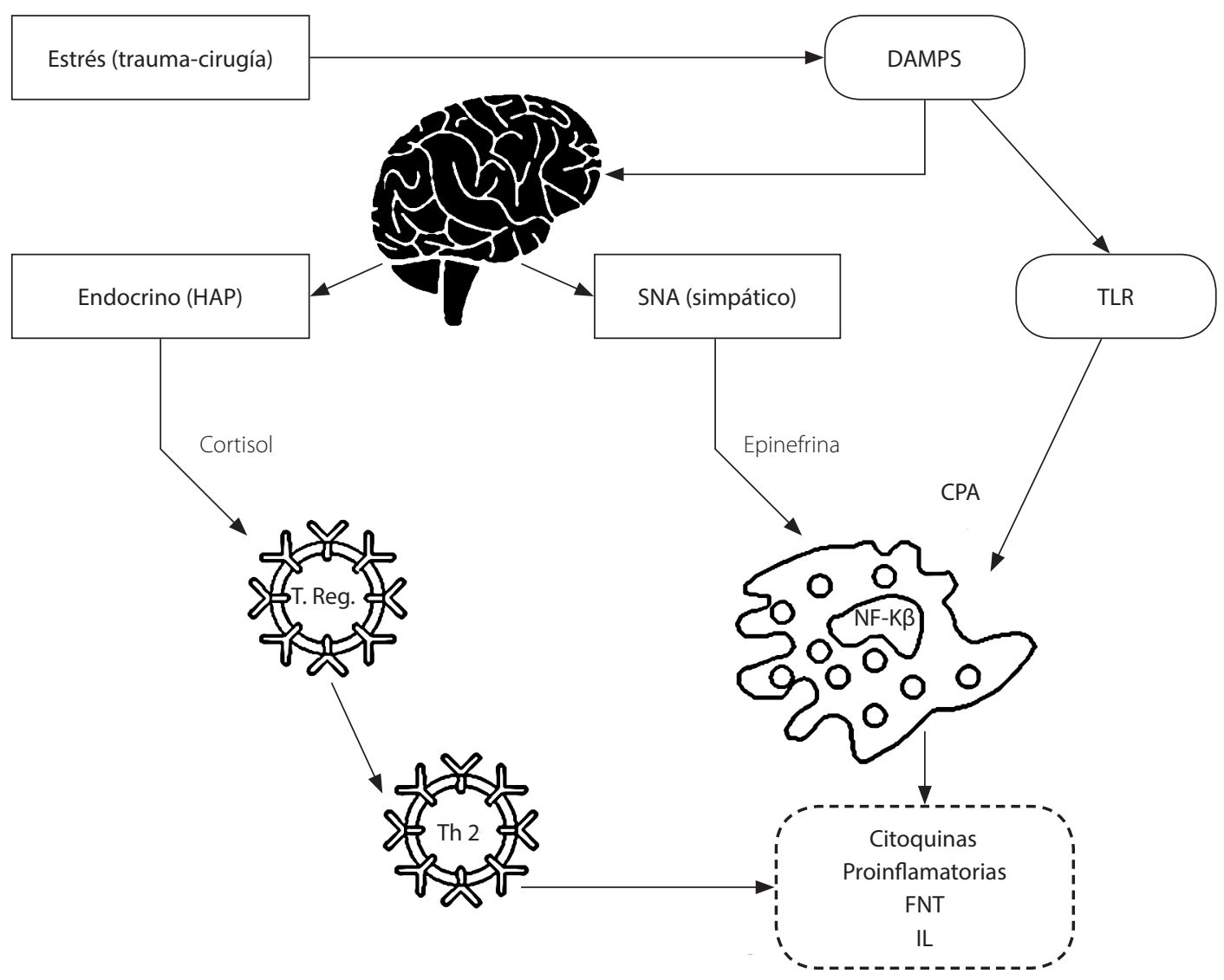

Figura 1. Respuesta inmune frente el trauma quirúrgico. DAMPS: patrones moleculares asociados a daño; TLR: toll like receptor; SNA: sistema nervioso autónomo; CPA: células presentadoras de antígeno; T. Reg.: linfocitos T reguladoras; Th 2: linfocitos T2; IL: interleuquinas; FNT: factor de necrosis. Adaptado y modificado de Marik PE et al. ${ }^{(6)}$.

bir las respuestas de los linfocitos $\mathrm{T}$ inducidas en linfocitos $\mathrm{T}$ activados $\mathrm{y}$ tienen mayor afinidad que el CD28 por las proteínas B7.

\section{b. Activación de la respuesta innata antiinflamatoria Th2}

La magnitud y la duración de las respuestas inmunitarias innatas están reguladas por varios mecanismos inhibidores que limitan el posible daño. Entre estas se encuentra la IL-10, la cual es una citocina producida por los macrófagos y las células dendríticas que inhibe la producción de varias citocinas inflamatorias producidas por las mismas células entre las que se incluye la IL-1, el TNF y la IL-12. Los fagocitos mononucleares producen un antagonista natural de la IL-1 que tiene una estructura homóloga a la citocinas y se une a los mismos receptores, pero carece de actividad biológica, de manera que funciona como un inhibidor competi- tivo de la IL-1, se llama antagonista del receptor para la IL-1 (IL-1RA).

Además existen numerosas vías de transmisión de señales reguladoras negativas que bloquean las señales activadoras generadas. Estos supresores de proteínas transmisoras de señales de citocinas (SOCS) son inhibidores de las vías de transmisión de las señales JAK-STAT ligadas a receptores de citocinas, las señales del TLR en los macrófagos y en las células dendríticas inducen la expresión de proteínas SOCS, que limitan las respuestas de estas células a citocinas exógenas como los interferones del tipo $\mathrm{I}^{(6,15)}$.

\section{c. Mediante la producción de antioxidantes enzimáticos}

Existen condiciones patológicas relacionadas con un estado conocido como estrés oxidativo, es decir, un aumento en las Especies Reactivas del Oxígeno 
(ERO) asociado a una disminución en los mecanismos de detoxificación de ellas. Entre los mecanismos de defensa existe una serie de antioxidantes tanto enzimáticos como no enzimáticos que contrarrestan el efecto de las ERO. Entre estos están la súper oxido dismutasa y la catalasa que permiten la dismutación del anión superóxido $\left(\mathrm{O}_{2}\right)$ a especies menos reactivas como el agua $\left(\mathrm{O}_{2} \mathrm{y} \mathrm{H}_{2} \mathrm{O}\right)$. En células de mamíferos existen dos tipos de SOD descritas: la enzima $\mathrm{Cu}-\mathrm{Zn}$ localizada en el citosol y la enzima Mn principalmente en mitocondria. Aunque esta última enzima está bajo control de procesos reguladores inducidos, por ejemplo, por Interleuquina 6 y los glucocorticoides, ambas enzimas catalizan la reacción de dismutación con la misma eficiencia.

\section{4. ¿Qué inmunonutrientes han demostrado beneficio en la administración perioperatoria?}

Se alcanzó consenso en considerar como inmunonutrientes con efecto benéfico los siguientes (Tabla 3):

1. Arginina. Los niveles altos en plasma se correlacionan con una síntesis elevada de colágeno; contribuye con una mejor utilización de la glucosa mediante la estimulación de insulina por el páncreas y mejora la sensibilidad de los tejidos a la misma. Aumenta la expresión de los receptores TCR de los linfocitos T con mejora de su función: la cicatrización ${ }^{(10,19)}$.

2. Nucleótidos. Son moléculas formadas por la unión covalente de una pentosa, una base nitrogenada y un grupo fosfato. Son los monómeros de los ácidos nucleicos (ADN y ARN). Sirven como transportadores de energía como moléculas libres (por ejemplo, el ATP o el GTP). Constituyen los sustratos metabólicos más abundantes para todos los organismos vivos. Las purinas proporcionan la energía necesaria y los cofactores para promover la supervivencia celular y la proliferación. Las purinas y sus derivados participan ampliamente en procesos biológicos que incluyen la respuesta inmune y las interacciones entre huésped y diferentes células.

3. Glutamina. Aminoácido condicionalmente esencial en situaciones críticas, donde las necesidades exceden la producción endógena del organismo. Durante el estrés catabólico (trauma, sepsis, quemaduras) es liberado, en forma rápida, desde los depósitos musculares y el suero. Es el principal nutriente usado por el enterocito. Presenta una acción importante en el tejido linfoide asociado al intestino, modulando la respuesta inmunológica local intestinal y sistémica. Produce estabilización del conteo de linfocitos, del porcentaje de CD4 activados, de linfocitos $\mathrm{T}$ ayudadores y los niveles de IL-2, los cuales se relacionan

Tabla 3. Principales inmunonutrientes $(10,11,16,36)$

\begin{tabular}{|l|l|}
\hline Arginina & $\begin{array}{l}\text { Aminoácido no esencial que se convierte en esencial durante estados de estrés y enfermedad crítica. Es un } \\
\text { substrato para células inmunitarias especialmente para la función linfocitaria. Es un precursor de óxido nítrico } \\
\text { e hidroxiprolina los cuales cumplen una función importante en la reparación del tejido conectivo. El déficit } \\
\text { de arginina se caracteriza por una respuesta inmune adaptativa deficiente secundaria a anormalidades en los } \\
\text { receptores de las células T. }\end{array}$ \\
\hline Glutamina & $\begin{array}{l}\text { Fuente energética para macrófagos, linfocitos y enterocitos. Participa en la señalización celular, mejora } \\
\text { la expresión de proteínas de choque térmico, previene la apoptosis, y disminuye la inflamación. La } \\
\text { suplementación de glutamina es esencial en estados catabólicos ya que las reservas de glutamina descienden } \\
\text { rápidamente. La deficiencia tiene como consecuencia la alteración de la función inmunológica y en la función } \\
\text { epitelial intestinal. }\end{array}$ \\
\hline $\begin{array}{l}\text { Ácidos grasos } \\
\text { omega-3 }\end{array}$ & $\begin{array}{l}\text { Tiene múltiples funciones antiinflamatorias como la reducción en la síntesis de eicosanoides proinflamatorios, } \\
\text { reducción en la interacciones adhesivas endoteliales de leucocito y plaquetas, inhibición de la expresión } \\
\text { génica inflamatoria y estimulación de la producción de glutatión, lo que puede causar disminución en la } \\
\text { oxidación durante la injuria. }\end{array}$ \\
\hline Nucleótidos & $\begin{array}{l}\text { Derivados de purinas y pirimidinas, participan en el desarrollo del sistema inmune, el crecimiento y } \\
\text { diferenciación tisular en especial en tejidos con recambio (turnover) rápido como la piel, células de la mucosa } \\
\text { intestinal, células de la médula ósea y linfocitos. Participan en la maduración, activación y proliferación de } \\
\text { linfocitos, estimulan la función fagocitaria de los macrófagos, modulan la respuesta de de hipersensibilidad } \\
\text { retardada, la respuesta contra los tumores y los trasplantes, la producción de inmunoglobulinas y la respuesta } \\
\text { a la infección. }\end{array}$ \\
\hline
\end{tabular}


con complicaciones sépticas del paciente con trauma múltiple, quirúrgico y quemado ${ }^{(10,19,29)}$.

4. Omega 3. Los ácidos grasos Omega 3 atenúan la producción de prostanglandinas inflamatorias y prostaciclinas. Los ácidos grasos eicosapentaenoico (EPA) y docosahexaenoico (DHA), regulan la función inmune, la producción de radicales libres y proporcionan los promotores del metabolismo de los eicosanoides. El DHA y EPA son precursores de resolvinas y protectinas, los cuales favorecen la resolución de la inflamación, por reducción e inhibición del transporte de células inflamatorias y mediadores en el sitio de injuria. Después de ser metabolizados a prostanglandinas antiinflamatorias y leucotrienos, suprimen RNA para la síntesis de interleuquina 1(IL-1) e inhiben la síntesis de factor de necrosis tumoral alfa (TNF-a), la proliferación de células $\mathrm{T}$ CD4 y de células Th1 ayudadoras, lo que determina un mecanismo de modulación de la respuesta inflamatoria.

5. Antioxidantes postoperatorios. Los AOX atenúan los cambios energéticos celulares, protegen la circulación microvascular, reducen la peroxidación lipídica, mejoran el gasto cardiaco, reducen la cantidad de volumen necesaria para la reanimación, disminuyen y optimizan el tiempo de cicatrización de una herida.

Ácido ascórbico (Vitamina C), cofactor enzimático y antioxidante. Disminuye la peroxidación lipídica y mantiene estables los niveles de glutatión peroxidasa $y$ de vitamina E. Los pacientes con sepsis, hemorragia, fallo multiorgánico, accidente cerebrovascular, lesión cerebral traumática o poscirugía cardíaca presentan bajas concentraciones de vitamina $\mathrm{C}$ en plasma, sugiriendo su consumo durante el estrés oxidativo.

Calciferol (Vitamina D), tiene un rol en la respuesta inmune innata contra bacterias, hongos y virus a través de la creación de péptidos antimicrobianos como las defensinas y la catelicidina. Estas proteínas preservan la integridad de las barreras en el tracto gastrointestinal, controlan las bacterias que usualmente residen en el tracto gastrointestinal y ayudan a resistir la invasión por otros patógenos. La hormona fisiológicamente activa también regula la ocludina, conexina y la actividad de la E-caderina, la cual es requerida para uniones estrechas, uniones gap y uniones adherentes en la mucosa intestinal ${ }^{(19,29)}$.

Selenio (Se), tiene un poder antioxidante e inmunomodulador. Forma parte del aminoácido selenocisteína, constituyendo las denominadas selenoproteínas, que funcionan como cofactor enzimático en múltiples reacciones biológicas. El descenso de selenio está asociado a mayor estrés oxidativo, disfunción orgánica múltiple, complicaciones infecciosas, $y$ el incremento de mortalidad en los pacientes en estado crítico.

Zinc ( $\mathrm{Zn})$, cofactor de numerosas metaloenzimas, deshidrogenasas y carboxipeptidasas implicadas en la síntesis de $\mathrm{ADN}$ y en diversos procesos metabólicos para los tejidos de rápido crecimiento. Se requiere homeostasis normal del zinc para un sistema inmune funcional, capacidad adecuada antioxidante, homeostasis de glucosa y cicatrización de heridas. La deficiencia de $\mathrm{Zn}$ se ha relacionado con involución tímica, reducción de la actividad de los linfocitos $\mathrm{T}$, macrófagos y de producción de inmunoglobulinas. Los requerimientos pueden aumentar por estrés metabólico (trauma, cirugía, quemaduras) y las pérdidas por fístulas o débitos gastrointestinales elevados. El exceso de $\mathrm{Zn}$ puede producir efectos adversos asociados con el deterioro del estado nutricional con respecto al $\mathrm{Cu}$ y $\mathrm{Fe}$, alteraciones en la respuesta inmune y reducción de las lipoproteínas de alta densidad.

Magnesio ( $\mathrm{Mg}$ ), tiene un rol en unión con el $\mathrm{Zn}$ de modulación de los niveles de TNF-a y expresión aumentada del factor beta de transformación del crecimiento (TGF-b), en el factor de crecimiento del endotelio vascular (VEGF) y en la isoforma $b$ del factor de crecimiento de fibroblastos (b-FGF). Se ha postulado que su unión con el $\mathrm{Zn}$ pueden resultar en modelos para la anastomosis del tracto gastrointestinal, en disminución de los niveles de mediadores inflamatorios y en reducción de la infiltración celular.

Otros AOX importantes son la Vitamina A, que optimiza la respuesta temprana a los procesos de inflamación, mejora la síntesis proteica y de colágeno, ayuda con la epitelización y función inmune; Vitamina $\mathrm{E}$, antioxidante con mecanismos similares al de otras moléculas de vitaminas liposolubles; Complejo-Vitamina B, apoyan la fase de proliferación con síntesis de proteínas y síntesis de colágeno; Cobre $(\mathrm{Cu})$, colaborador en la síntesis de elastina y colágeno, Hierro $(\mathrm{Fe})$ favorece la cicatrización de herida, transporte de $\mathrm{O}_{2}$ y ayudar en la función inmune.

6. Simbióticos (denominación para combinación de prebióticos y probióticos). Son organismos vivos que confieren un beneficio en la salud del huésped por servir de profilaxis de infecciones postoperatorias previniendo el sobrecrecimiento bacteriano, 
mejorando la función de barrera intestinal y modulando la respuesta inmune. Los prebióticos son la fibra no digerible que estimula el crecimiento o la actividad de los probióticos. Los probióticos tienen capacidad de prevenir las infecciones en pacientes sometidos a cirugía gastrointestinal y en trasplante hepático, y disminuyen las infecciones por prevención de la translocación por estabilización de la barrera intestinal. Además, estimulan el crecimiento epitelial, la secreción mucosa y la motilidad y optimizan la inmunidad innata por la estimulación de la producción de la Inmunoglobulina A secretora, estimulación de neutrófilos y por reducción de las citocinas inflamatorias ${ }^{(19,29,77)}$.

También se obtuvo consenso en considerar como inmunonutrientes carentes de beneficio, los siguientes:

1. El uso de un inmunonutriente de forma aislada y sin relación con los demás y dentro de una fórmula de nutrición específica, pues cuando se han administrado de forma aislada, no se han encontrado efectos estadísticamente significativos en los desenlaces.

2. Los productos denominados fitoterapéuticos.

3. Los productos que se promocionan como efectivos en ámbitos de ejercicio y deportes.

\section{5. ¿Los fórmulas inmunoenriquecidas son seguras en cirugía?}

Se obtuvo consenso al considerar que el uso de fórmulas inmunoenriquecidas son seguras en el perioperatorio, teniendo en cuenta una adecuada selección del paciente y siguiendo las recomendaciones de tiempo, vía y dosis de administración ${ }^{(16,17)}$.

\section{6. ¿En qué pacientes quirúrgicos considera que existe beneficio en el uso de inmunonutrición perioperatoria?}

Para responder esta pregunta se diseñó una jerarquía estructurada de los pacientes potenciales (Figura 2).

Se obtuvo consenso respecto a un beneficio clínico de la inmunonutrición (fórmulas enriquecidas con inmunonutrientes -arginina, omega 3 y ribonucleótidos) en los siguientes grupos de pacientes quirúrgicos ${ }^{(2-4,17-54)}$ :

1. Los pacientes en estado de malnutrición que serán sometidos a cirugía mayor de cabeza y cuello (cirugías que implican resecciones amplias y reconstrucción con colgajo loco regional o microquirúrgico) y gastrointestinal mayor (cirugía gástrica, hepatobiliopancreática, colónica que requiere resección y anastomosis) independiente del momento de la administración (pre o postoperatoria), del tipo de cirugía (electiva o urgente) o de la condición que indica la cirugía (enfermedad oncológica o no oncológica). Si la cirugía es electiva, la recomendación es iniciar la inmunonutrición en el preoperatorio.

2. Pacientes con trauma mayor y quemaduras severas, independiente de su estado nutricional previo.

Se obtuvo consenso respecto a la ausencia de beneficio clínico de la inmunonutrición (fórmulas enriquecidas con inmunonutrientes -arginina, omega 3 y ribonucleótidos) en los siguientes grupos de pacientes quirúr$\operatorname{gicos}^{(2-4,17-54)}$ :

1. En postoperatorio electivo o no electivo de paciente bien nutrido con enfermedad no oncológica que fue sometido a cirugía mayor de cabeza y cuello (cirugías que implican resecciones amplias y reconstrucción con colgajo loco-regional o microquirúrgico) y gastrointestinal mayor (cirugía gástrica, hepatobilopancreática, colónica que requiere resección y anastomosis.

2. En postoperatorio electivo de pacientes bien nutrido con enfermedad oncológica que fue sometido a cirugía mayor de cabeza y cuello (cirugías que implican resecciones amplias y reconstrucción con colgajo loco-regional o microquirúrgico) y gastrointestinal mayor (cirugía gástrica, hepatobilopancreática, colónica que requiere resección y anastomosis.

3. Pacientes con sepsis severa.

4. Pacientes de cirugía cardiaca de alto riesgo, según el Euro SCORE II mayor a 5.

No se obtuvo consenso respecto a definir el beneficio clínico de la inmunonutrición (fórmulas enriquecidas con inmunonutrientes -arginina, omega 3 y ribonucleótidos) en los siguientes grupos de pacientes quirúr$\operatorname{gicos}^{(3,4,17-42,55-63):}$

1. En el preoperatorio electivo del paciente bien nutrido con enfermedad oncológica o no oncológica que será sometido a cirugía mayor de cabeza y cuello (cirugías que implican resecciones amplias y reconstrucción con colgajo loco-regional o microquirúrgico) y gastrointestinal mayor (cirugía gástrica, hepatobiliopancreática, colónica) que requieran resección y anastomosis.

2. En el postoperatorio urgente del paciente bien nutrido con enfermedad oncológica que fue sometido a cirugía mayor de cabeza y cuello (cirugías que implican resecciones amplias y reconstrucción con colga- 


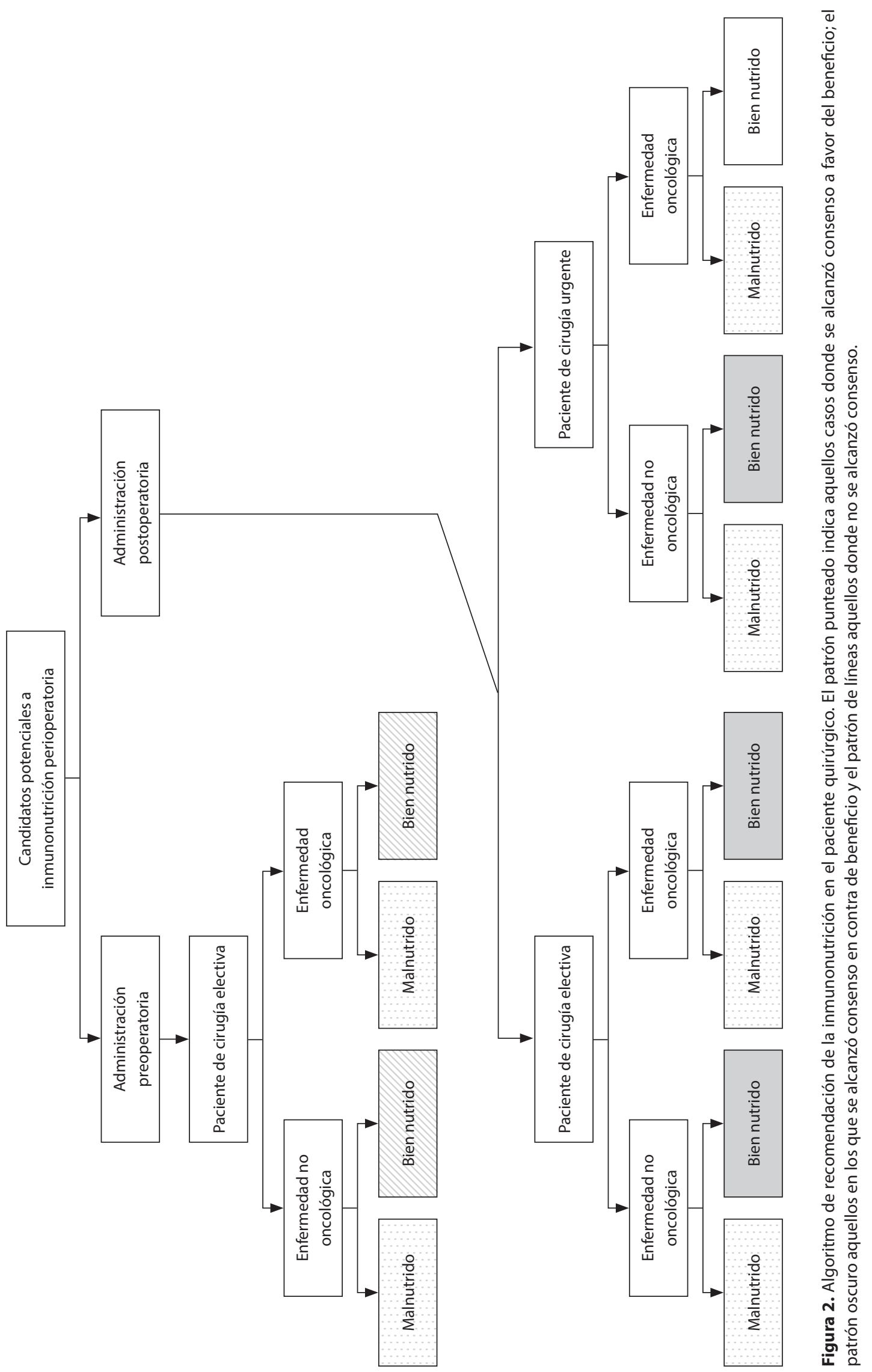


jo loco-regional o microquirúrgico) y gastrointestinal mayor (cirugía gástrica, hepatobiliopancreática, colónica que requiere resección y anastomosis).

3. En cirugía de trasplante (hígado, intestino, páncreas, corazón, pulmón).

\section{7. ¿En qué pacientes quirúrgicos está contraindicada la Inmunonutrición?}

Se obtuvo consenso en que el uso de inmunonutrición está contraindicada en pacientes con sepsis severa, dado que uno de los componentes es la arginina, la cual es un sustrato del óxido nítrico, y en este subgrupo de pacientes se incrementaría la mortalidad ${ }^{(49,54,64-67)}$.

\section{8. ¿Cuánto tiempo previo a la cirugía se debe iniciar la terapia de inmunonutrición?}

Se obtuvo consenso respecto al tiempo de administración así ${ }^{(21,25,26,29,47,48,53,65,68-71)}$ :

1. En el preoperatorio se debe iniciar entre 5 y 7 días previos a la cirugía

2. En el preoperatorio, 10 o más días no han demostrado mejoría en los desenlaces clínicos.

\section{9. ¿Cuál debe ser la dosis necesaria para el beneficio planteado en la inmunonutrición preoperatoria?}

Se obtuvo consenso respecto a las dosis así $i^{(21,25,26,29,47,}$ 48,53,65,68-71):

1. Para arginina, la dosis recomendada debe ser de 6-18 g / día

2. Para ácidos grasos omega-3, la dosis recomendada debe ser 1-3 g/día

3. Menos de $6 \mathrm{~g} /$ día de arginina no ofrece beneficio cuando se compara con fórmulas estándar

4. No se conocen los efectos benéficos con dosis de arginina mayores a $18 \mathrm{~g} /$ día.

Se discutió que la administración por vía oral de estas cantidades es difícil de alcanzar dado el volumen necesario y la palatabilidad. Debe entonces, garantizarse una vía de administración enteral por sonda, cuando el paciente no alcanza las metas por vía oral.

\section{0. ¿Cuál es la ruta de administración recomendada?}

Se obtuvo consenso respecto a la vía así $i^{(21,25,26,29,47,48,}$ $53,65,68-71)$ :
1. La vía recomendada es la vía oral o enteral por sonda

2. No existe presentación para uso parenteral disponible que pueda recomendarse.

\section{1. ¿Qué otras medidas no farmacológicas deberían ser usadas para mejorar los resultados cuando se emplea inmunonutrición preoperatoria?}

Se obtuvo consenso respecto a las medidas farmacológicas asociadas a la inmunonutrición así:

1. Se deben iniciar maniobras para el control de la hiperglicemia

2. Se deben asociar medidas para disminuir el peso en obesos Grado II y III (dieta y ejercicio)

3. Se deben iniciar maniobras para disminuir el tabaquismo

4. Se deben implementar estrategias para recuperación postoperatoria (Protocolo ERAS)

5. No se conocen los efectos de las dietas ricas en antioxidantes u omega-3 aislado, por lo cual no es posible realizar una recomendación.

\section{2. ¿Qué factores hacen costo-efectivas las dietas inmunomuduladoras?}

Se obtuvo consenso respecto a los factores que hacen costo-efectivas las dietas inmunomuduladoras asi (29,72-76):

1. En paciente malnutridos hay disminución en los días de estancia hospitalaria, días de estancia en UCI e infecciones asociadas al cuidado de la salud.

2. No existe información acerca del impacto en otros desenlaces.

Se consideró que la disponibilidad de fórmulas en el país y su precio actual junto con la falta de adherencia al tratamiento pueden afectar la costo-efectividad.

\section{3. ¿Qué dificultades existen en Colombia para la implementación de una terapia nutricional como esta?}

Se obtuvo consenso respecto a las dificultades de implementación así ${ }^{(12)}$ :

1. Existe un desconocimiento importante de las potenciales ventajas clínicas del uso de inmunonutrición

2. Hay una ausencia de protocolos clínicos estandarizados para el uso de la inmunonutrición

3. Su administración rutinaria requiere áreas físicas específicas para preparación que no existen en todas las instituciones 
4. La disponibilidad de fórmulas en el país es limitada

5. Los costos actuales de las fórmulas son muy altos

6. Las características de las fórmulas en relación con su palatabilidad y tolerancia son pobres, lo que hace que la administración oral sea difícil. Esto determina una pérdida de adherencia al tratamiento que compromete las potenciales ventajas clínicas.

\section{4. ¿Qué intervenciones reemplazaría el uso de la inmunonutrición en los pacientes en que se demuestre beneficio?}

Se obtuvo consenso respecto a que el uso rutinario en los casos enunciados previamente reemplaza el uso de la nutrición estándar y solo tendría un aumento marginal en el costo del tratamiento.

\section{CONCLUSIONES}

Se han planteado recomendaciones respecto al uso de inmunonutrición en el paciente con patología quirúrgica en el periodo perioperatorio, las cuales han sido adaptadas a nuestra condiciones locales como país, en cuanto a la disponibilidad, costos, barreras y beneficios demostrados en los subgrupos de pacientes.

Este consenso permite unificar conceptos, y brinda unos parámetros basados en la literatura y la experiencia de los expertos para un uso adecuado de inmunonutrición en el periodo perioperatorio.

\section{Financiación}

El presente consenso fue financiado por la ACNC.

\section{Conflicto de intereses}

Los autores declaran no tener conflicto de intereses en relación al consenso.

\section{Referencias bibliográficas}

1. Meakins JL. Host defense mechanisms in surgical patients: effect of surgery and trauma. Acta Chir Scand Suppl. 1989;50: 43- 53.

2. Heys SD, Walker LG, Smith I, Eremin O. Enteral nutritional supplementation with key nutrients in patients with critical illness and cancer: a meta-analysis of randomized controlled clinical trials. Ann Surg. 1999;229(4): 467- 77.

3. Stableforth WD, Thomas S, Lewis SJ. A systematic review of the role of immunonutrition in patients undergoing surgery for head and neck cancer. Int J Oral Maxillofac Surg. 2009;38(2):103-10.
4. Zhang Y, Gu Y, Guo T, Li Y, Cai H. Perioperative immunonutrition for gastrointestinal cancer: a systematic review of randomized controlled trials. Surg Oncol. 2012;21(2):e87-95.

5. Senkal M, Zumtobel V, Bauer KH, Marpe B, Wolfram G, Frei A, et al. Outcome and Cost-effectiveness of Perioperative Enteral Immunonutrition in Patients Undergoing Elective Upper Gastrointestinal Tract Surgery. A Prospective Randomized Study. Arch Surg. 1999;134(12):1309-16.

6. Marik PE, Flemmer M. The immune response to surgery and trauma: Implications for treatment. J Trauma Acute Care Surg. 2012;73(4):801-808.

7. Veterans Affairs Total Parenteral Nutrition Cooperative Study Group. Perioperative total parenteral nutrition in surgical patients. N Engl J Med.1991;325(8):525-32.

8. Heyland DK. Immunonutrition in the critically ill patient: putting the cart before the horse? Nutr Clin Pract. 2002;17(5):267-72.

9. Heyland DK, Novak F. Immunonutrition in the critically ill patient: more harm than good? JPEN J Parenter Enteral Nutr. 2001;25(2 Suppl):S51-56.

10. Koretz RL. Immunonutrition: fact, fantasy, and future. Curr Gastroenterol Rep. 2002;4(4):332-7.

11. McCowen KC, Bistrian BR. Immunonutrition: problematic or problem solving? Am J Clin Nutr. 2003;77(4):764-70.

12. Bermudez C, Sanabria Á, Vega V. Soporte nutricional del paciente adulto. Resultados de la reunión de consenso de la Asociación Colombiana de Nutrición Clínica. Rev Colomb Cir. 2013;28(2):15.

13. Lin E, Calvano SE, Lowry SF. Cytokine response in abdominal surgery. En: Schein M, Wise L, eds. Cytokines and the Abdominal Surgeon. Austin: Landes,1998. p.17-34.

14. Uauy R. Academic-industry partnerships in addressing nutrition--[infection-immunity-inflammation] interactions. $\mathrm{Br} \mathrm{J}$ Nutr. 2007;98 Suppl 1:S17-23.

15. Suchner U, Kuhn KS, Furst P. The scientific basis of immunonutrition. Proc Nutr Soc. 2000;59(4):553-63.

16. Kudsk KA. Immunonutrition in surgery and critical care. Annu Rev Nutr. 2006;26:463-79.

17. Zheng Y, Li F, Qi B, Luo B, Sun H, Liu S, et al. Application of perioperative immunonutrition for gastrointestinal surgery: a meta-analysis of randomized controlled trials. Asia Pac J Clin Nutr. 2007;16(Suppl) 1:253-7.

18. Braga M, Gianotti L, Radaelli G, Vignali A, Mari G, Gentilini $\mathrm{O}$, et al. Perioperative immunonutrition in patients undergoing cancer surgery: results of a randomized double-blind phase 3 trial. Arch Surg. 1999;134(4):428-33.

19. Cerantola Y, Hubner M, Grass F, Demartines N, Schäfer $\mathrm{M}$. Immunonutrition in gastrointestinal surgery. $\mathrm{Br} \mathrm{J}$ Surg. 2011;98(1):37-48.

20. de Luis DA, Izaola O, Aller R, Cuellar L, Terroba MC. A randomized clinical trial with oral Immunonutrition (omega3- 
enhanced formula vs. arginine-enhanced formula) in ambulatory head and neck cancer patients. Ann Nutr Metab. 2005;49(2):95-9.

21. Fujitani K, Tsujinaka T, Fujita J, Miyashiro I, Imamura H, Kimura $\mathrm{Y}$, et al. Prospective randomized trial of preoperative enteral immunonutrition followed by elective total gastrectomy for gastric cancer. Br J Surg. 2012;99(5):621-9.

22. Gade J, Levring T, Hillingso J, Hansen CP, Andersen JR. The Effect of Preoperative Oral Immunonutrition on Complications and Length of Hospital Stay After Elective Surgery for Pancreatic Cancer--A Randomized Controlled Trial. Nutr Cancer. 2016;68(2):225-33.

23. Helminen $\mathrm{H}$, Raitanen $\mathrm{M}$, Kellosalo J. Immunonutrition in elective gastrointestinal surgery patients. Scand J Surg. 2007;96(1):46-50.

24. Ida S, Hiki N, Cho H, Sakamaki K, Ito S, Fujitani K, et al. Randomized clinical trial comparing standard diet with perioperative oral immunonutrition in total gastrectomy for gastric cancer. Br J Surg. 2017;104(4):377-83.

25. Luo Z, Wang J, Zhang Z, Li H, Huang L, Qiao Y, et al. Efficacy of Early Enteral Immunonutrition on Immune Function and Clinical Outcome for Postoperative Patients With Gastrointestinal Cancer. JPEN J Parenter Enteral Nutr. 2017:148607117715439.

26. Marano L, Porfidia R, Pezzella M, Grassia M, Petrillo M, Esposito G, et al. Clinical and immunological impact of early postoperative enteral immunonutrition after total gastrectomy in gastric cancer patients: a prospective randomized study. Ann Surg Oncol. 2013;20(12):3912-3918.

27. Moskovitz DN, Kim YI. Does perioperative immunonutrition reduce postoperative complications in patients with gastrointestinal cancer undergoing operations? Nutr Rev. 2004;62(11):443-7.

28. Mudge L, Isenring E, Jamieson GG. Immunonutrition in patients undergoing esophageal cancer resection. Dis Esophagus. 2011;24(3):160-5.

29. Hegazi R, Hustead D, Evans D. Preoperative Standard Oral Nutrition Supplements vs Immunonutrition: Results of a Systematic Review and Meta Analysis. Am Coll Surg. Vol. 219, No. 5, November 2014.

30. Sodergren MH, Jethwa P, Kumar S, Duncan HD, Johns T, Pearce CB. Immunonutrition in patients undergoing major upper gastrointestinal surgery: a prospective double-blind randomised controlled study. Scand J Surg. 2010;99(3):153-161.

31. Song GM, Liu XL, Bian W, Wu J, Deng YH, Zhang H, et al. Systematic review with network meta-analysis: comparative efficacy of different enteral immunonutrition formulas in patients underwent gastrectomy. Oncotarget. 2017;8(14):23376-8.

32. Song GM, Tian X, Liang H, Yi LJ, Zhou JG, Zeng Z, et al. Role of Enteral Immunonutrition in Patients Undergoing Surgery for Gastric Cancer: A Systematic Review and Meta-Analysis of Randomized Controlled Trials. Medicine (Baltimore). 2015;94(31):e1311.
33. Song GM, Tian X, Zhang L,Ou YX, Yi LJ, Shuai T, et al. Immunonutrition Support for Patients Undergoing Surgery for Gastrointestinal Malignancy: Preoperative, Postoperative, or Perioperative? A Bayesian Network Meta-Analysis of Randomized Controlled Trials. Medicine (Baltimore). 2015;94(29):e1225.

34. Wong CS, Aly EH. The effects of enteral immunonutrition in upper gastrointestinal surgery: A systematic review and metaanalysis. Int J Surg. 2016;29:137-50.

35. Xu J, Zhong Y, Jing D, Wu Z. Preoperative enteral immunonutrition improves postoperative outcome in patients with gastrointestinal cancer. World J Surg. 2006;30(7):1284-9.

36. Yildiz SY, Yazicioglu MB, Tiryaki C, Ciftci A, Boyacioglu Z. The effect of enteral immunonutrition in upper gastrointestinalsurgery for cancer: a prospective study. Turk J Med Sci. 2016;46(2):393-400.

37. Falewee MN, Schilf A, Boufflers E, Cartier C, Bachmann $\mathrm{P}$, Pressoir $\mathrm{M}$, et al. Reduced infections with perioperative immunonutrition in head and neck cancer: exploratory results of a multicenter, prospective, randomized, double-blind study. Clin Nutr. 2014;33(5):776-84.

38. Machon C, Thezenas S, Dupuy AM, Assenat E, Michel F, Mas E, et al. Immunonutrition before and during radiochemotherapy: improvement of inflammatory parameters in head and neck cancer patients. Supportive Care Cancer. 2012;20(12):3129-35.

39. Riso S, Aluffi P, Brugnani M, Farinetti F, Pia F, D’Andrea F. Postoperative enteral immunonutrition in head and neck cancer patients. Clin Nutr. 2000;19(6):407-12.

40. Sorensen D, McCarthy M, Baumgartner B, Demars S. Perioperative immunonutrition in head and neck cancer. Laryngoscope. 2009;119(7):1358-64.

41. Turnock A, Calder PC, West AL, Izzard M, Morton RP, Plank LD. Perioperative immunonutrition in well-nourished patients undergoing surgery for head and neck cancer: evaluation of inflammatory and immunologic outcomes. Nutrients. 2013;5(4):1186-99.

42. Vasson MP, Talvas J, Perche O, Dillies AF, Bachmann P, Pezet $\mathrm{D}$, et al. Immunonutrition improves functional capacities in head and neck and esophageal cancer patients undergoing radiochemotherapy: a randomized clinical trial. Clin Nutr.. 2014;33(2):204-10.

43. Farreras N, Artigas V, Cardona D, Rius X, Trias M, Gonzalez JA. Effect of early postoperative enteral immunonutrition on wound healing in patients undergoing surgery for gastric cancer. Clin Nutr. 2005;24(1):55-65.

44. Kamocki Z, Matowicka-Karna J, Gryko M, Zareba K, Kedra B, Kemona $\mathrm{H}$. The effect of perioperative immunonutrition on the phagocytic activity of blood platelets in advanced gastric cancer patients. Clin Dev Immunol. 2013;2013:435672.

45. Klek S, Szybinski P, Szczepanek K. Perioperative immunonutrition in surgical cancer patients: a summary of a decade of research. World J Surg. 2014;38(4):803-12. 
46. Mabvuure NT, Roman A, Khan OA. Enteral immunonutrition versus standard enteral nutrition for patients undergoing oesophagogastric resection for cancer. Int J Surg. 2013;11(2):122-7.

47. Qiang H, Hang L, Shui SY. The curative effect of early use of enteral immunonutrition in postoperative gastric cancer: a meta-analysis. Minerva Gastroenterol Dietol. 2017;63(3):285-92.

48. Bastian L, Weimann A. Immunonutrition in patients after multiple trauma. Br J Nutr. 2002;87(Suppl 1): S133-134.

49. Beale RJ, Bryg DJ, Bihari DJ. Immunonutrition in the critically ill: a systematic review of clinical outcome. Crit Care Med. 1999;27(12):2799-805.

50. Calder PC. Immunonutrition in surgical and critically ill patients. Br J Nutr. 2007;98(Suppl 1):S133-9.

51. Chuntrasakul C, Siltham S, Sarasombath S, Sittapairochana C, Leowattana W, Chockvivatanavanit S, et al. Comparison of a immunonutrition formula enriched arginine, glutamine and omega-3 fatty acid, with a currently high-enriched enteral nutrition for trauma patients. J Med Assoc Thai. 2003;86(6):552-61.

52. Li SL, Xu YH, Wang X, Liu XF, Zhao L. Effects of enteral immunonutrition on immune function in patients with multiple trauma. World J Emerg Med. 2011;2(3):206-9.

53. Lorenz KJ, Schallert R, Daniel V. Immunonutrition - the influence of early postoperative glutamine supplementation in enteral/parenteral nutrition on immune response, wound healing and length of hospital stay in multiple trauma patients and patients after extensive surgery. GMS Interdiscip Plast Reconstr Surg DGPW. 2015;4:Doc15.

54. Marik PE, Zaloga GP. Immunonutrition in critically ill patients: a systematic review and analysis of the literature. Intensive Care Med. 2008;34(11):1980-90.

55. Alexander JW. Role of immunonutrition in reducing complications following organ transplantation. Transplant Proc. 2000;32(3):574-5.

56. Alexander JW. Immunonutrition in kidney and liver transplant recipients. Exp Clin Transplant. 2014;12(Suppl 1):34-37.

57. Bharadwaj S, Rivas JM, Jevenn A, Hanouneh I, Steiger E. Immunonutrition in patients undergoing liver transplantation: Too premature to draw conclusions. Hepatology. 2016;63(5):1741-2.

58. Kota $H$, Chamberlain RS. Immunonutrition Is Associated With a Decreased Incidence of Graft-Versus-Host Disease in Bone Marrow Transplant Recipients: A Meta-Analysis. JPEN J Parenter Enteral Nutr. 2017;41(8):1286-92.

59. Lei Q, Wang X, Zheng H, Bi J, Tan S, Li N. Peri-operative immunonutrition in patients undergoing liver transplantation: a meta-analysis of randomized controlled trials. Asia Pac J Clin Nutr. 2015;24(4):583-90.

60. Nagata S, Shirabe K, Sugimachi K, Ikegami T, Yoshizumi T, Uchiyama $\mathrm{H}$, et al. Pilot study of preoperative immunonu- trition with antioxidants in living donor liver transplantation donors. Fukuoka Igaku Zasshi. 2013;104(12):530-8.

61. Nickkholgh A, Schneider H, Encke J, Buchler MW, Schmidt J, Schemmer P. PROUD: Effects of preoperative long-term immunonutrition in patients listed for liver transplantation. Trials. 2007;8:20.

62. Plank LD, Mathur S, Gane EJ, Peng SL, Gillanders LK, Mcllroy K, et al. Perioperative immunonutrition in patients undergoing liver transplantation: a randomized double-blind trial. Hepatology. 2015;61(2):639-47.

63. Plank LD, McCall JL, Gane EJ, Rafique M, Gillanders LK, Mcllroy K, et al. Pre- and postoperative immunonutrition in patients undergoing liver transplantation: a pilot study of safety and efficacy. Clin Nutr. 2005;24(2):288-96.

64. Bertolini G, Iapichino G, Radrizzani D, Facchini R, Simini B, Bruzzone P, et al. Early enteral immunonutrition in patients with severe sepsis: results of an interim analysis of a randomized multicentre clinical trial. Intensive Care Med. 2003;29(5):834-40.

65. Briassoulis G, Filippou O, Hatzi E, Papassotiriou I, Hatzis T. Early enteral administration of immunonutrition in critically ill children: results of a blinded randomized controlled clinical trial. Nutrition. 2005;21(7-8):799-807.

66. Heyland DK, Samis A. Does immunonutrition in patients with sepsis do more harm than good? Intensive Care Med. 2003;29(5):669-71.

67. Knight DJW. Immunonutrition: increased mortality is associated with immunonutrition in sepsis. BMJ. 2003;327(7416):682-683.

68. Alivizatos V, Athanasopoulos P, Makris N, Karageorgos N. Early postoperative glutamine-supplemented parenteral nutrition versus enteral immunonutrition in cancer patients undergoing major gastrointestinal surgery. J BUON. 2005;10(1):119-22.

69. Atkinson S, Sieffert E, Bihari D. A prospective, randomized, double-blind, controlled clinical trial of enteral immunonutrition in the critically ill. Guy's Hospital Intensive Care Group. Crit Care Med. 1998;26(7):1164-72.

70. Bharadwaj S, Trivax B, Tandon P, Alkam B, Hanouneh I, Steiger E. Should perioperative immunonutrition for elective surgery be the current standard of care? Gastroenterol Rep (Oxf). 2016;4(2):87-95.

71. Radrizzani D, Bertolini G, Facchini R, Simini B, Bruzzone P, Zanforlin G, et al. Early enteral immunonutrition vs. parenteral nutrition in critically ill patients without severe sepsis: a randomized clinical trial. Intensive Care Med. 2006;32(8):1191-8.

72. Banerjee S, Garrison LP, Danel A, Ochoa Gautier JB, Flum DR. Effects of arginine-based immunonutrition on inpatient total costs and hospitalization outcomes for patients undergoing colorectal surgery. Nutrition. 2017;42:106-13.

73. Braga M, Gianotti L. Preoperative immunonutrition: costbenefit analysis. JPEN J Parenter Enteral Nutr. 2005;29(1 Suppl):S57-61. 
74. Gianotti L, Braga M, Frei A, Greiner R, Di Carlo V. Health care resources consumed to treat postoperative infections: cost saving by perioperative immunonutrition. Shock. 2000;14(3):325-30.

75. dos Reis AM, Kabke GB, Fruchtenicht AVG, Barreiro TD, Moreira LF. Cost-Effectiveness of Perioperative Immunonutrition in Gastrointestinal Oncologic Surgery: A Systematic Review. Arq Bras Cir Dig. 2016;29(2):121-5.
76. Senkal M, Mumme A, Eickhoff U, Geier B, Späth G, Wulfert $\mathrm{D}$, et al. Early postoperative enteral immunonutrition: clinical outcome and cost-comparison analysis in surgical patients. Crit Care Med. 1997;25(9):1489-96.

77. Zhiping Y, Qiong W, Yunfang L, Daiming F. Effect of Perioperative Probiotics and Synbiotics on Postoperative Infections After Gastrointestinal Surgery: A Systematic Review With Meta-Analysis. JPEN. 2017;41(6):1051-1062. 